\title{
Integral Field Spectrography of the double AGN merging system Markarian 463
}

\author{
E. Chatzichristou $(1),(2)$
}

(1) Sterrewacht Leiden, P.B. 9513, 2300 RA Leiden, The Netherlands

(2) European Southern Observatory, La Silla, Chile

\begin{abstract}
Markarian 463, a system of 2 interacting/merging galaxies ( $\mathrm{E}$ and $\mathrm{W}$ ) has been observed with SILFID at CFHT in sub-arcsec resolution conditions. We found that both nuclei are $A G N s$, with the $W$ component being a Sy 2 or LINER and the E component, hitherto known to be a "hidden" Seyfert 1, having essentially Sy 2 characteristics. The bipolar collimated emission originating from the $E$ nucleus is a conspicuous feature in the $[O I I I]_{5007}$ and $H \alpha$ reconstructed images, as well as in the $V-R$ color map. In velocity maps some previously undetected kinematical features are detected, most notably a sharp velocity gradient (more than $300 \mathrm{~km} \mathrm{~s}^{-1}$ ) within 2" $\mathrm{NE}$ of the E nucleus. In addition, in the direct imaging mode a bright optical jet extending $\sim 1.2^{\prime \prime} \mathrm{S}$ from the E nucleus and a fuzzy blue component $0.8^{\prime \prime} \mathrm{W}$ of the W nucleus were seen. Both were also discovered on HST images. Detailed ionization maps reveal individual regions with distinct physical conditions, which constrain the mechanism(s) responsible for the ionization of the gas.
\end{abstract}

\section{Introduction : The Mrk 463 system}

Although the origin of nuclear activity in galaxies is still not fully understood, most observational data can be reproduced by the black hole - accretion disk model. In this model of AGN, galaxy interaction and merging is an efficient mechanism for fuelling the central black hole, with gas supplied by the host galaxy, the interacting companion, or both (Roos 1981, Norman and Silk 1983). In order to further investigate how interaction and merging of galaxies can trigger their nuclear activity, we selected a sample of active galaxies with disturbed morphologies and/or multiple nuclei. Mrk 463 was one of the systems we observed in bidimensional spectroscopy as well as in direct imaging mode.

Optically, Mrk 463 is a complex system containing two central condensations, the $\mathrm{E}$ and $\mathrm{W}$ nuclei, separated by $\sim 4^{\prime \prime}(\simeq 4 \mathrm{Kpc}$ for $\mathrm{z} \simeq 0.05)$, embedded in a common diffuse body with prominent tidal tails. The $E$ nucleus shows a typical Seyfert 2 spectrum (strong, blue-asymmetric, broad-based lines) but polarization studies have shown clear signatures of a "hidden" Seyfert 1 nucleus (Miller and Goodrich, 1987 and 1990; Mazzarella et al. 1991). Hutchings and Neff (1989) discovered a linear $[\mathrm{OIII}]_{5007}$ feature centered on this nucleus, extending $\sim 20^{\prime \prime}$ in the N-S direction. It is associated with a similar radio structure (Neff and Ulvestad 1988, Mazzarella et al. 1991). Recently, a one-sided linear 
optical jet, aligned with the $[O I I I] /$ radio axis and dominated by continuum light, has been discovered on HST images (Uomoto et al., 1993). The nature of the west nucleus still remains unclear; it has been classified as either a Seyfert 2, a LINER, or a luminous starburst nucleus.

In our study we intend to clarify two points concerning Mrk 463

- Physical conditions and ionization mechanism(s) in the emitting regions.

- Global dynamical picture of the system.

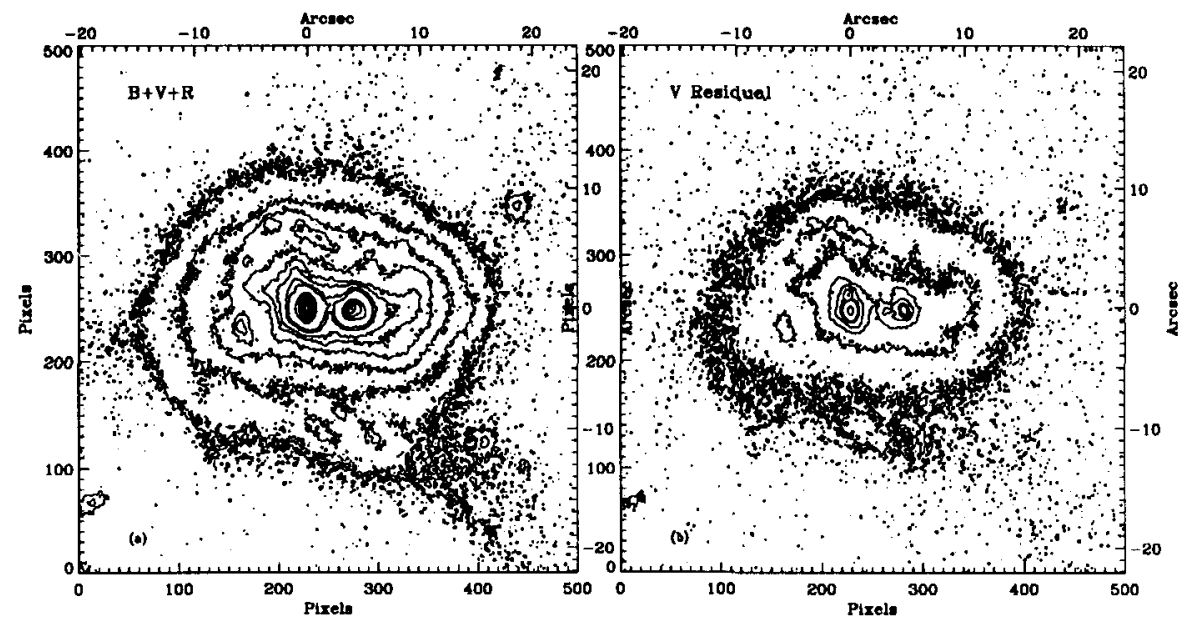

Figure 1. - a) Superposition of the B,V and R exposures on Mrk 463 (see text); - b) Residuals in V after subtraction of the two nuclei

\section{Observations with SILFID}

In February/March 1992, we observed Mrk 463 with SILFID at the CFH telescope (Mauna Kea, Hawaii) in good seeing conditions $\left(\sim 0.8^{\prime \prime}\right)$. The detector was a $2048 \times 2048 \mathrm{CCD}$ (pixel size $15 \mu \mathrm{m}$ ). The ARGUS mode for integral field spectroscopy (cf. review paper by Vanderriest elsewhere in this volume) was used in two configurations: $1.4^{\prime \prime} /$ fibre (whole system) and $0.33^{\prime \prime} /$ fibre (E nucleus) and with two grisms that covered the spectral range 4400-8000 $\AA$. In addition, we obtained $B, V$ and $R$ images covering $\sim 4.5^{\prime}$ (reduced to $1500 \times 1500$ pixels of $\left.0.178^{\prime \prime}\right)$. A detailed description of the data processing can be found in a forthcoming paper (Chatzichristou and Vanderriest 1994, hereafter CV). Individual spectra (about 70 for each grism), corresponding to 1 fibre or to the average of a few fibres, have been processed for determining several parameters of the most important emission lines: (a) corrected line fluxes; (b) central velocities at 3 levels, namely $80 \%, 50 \%, 20 \%$ of the peak intensity of the line; (c) full widths at the same 3 levels and (d) the $20 \%$ line asymmetries. We also reconstructed intensity and velocity maps by inversion of the geometrical transformation defined by the fibre bundle. 


\section{Results}

\subsection{Photometry}

Table 1. Photometric data

\begin{tabular}{|c|c|c|c|}
\hline Object & $\mathrm{V}$ & (B-V) & (V-R) \\
\hline E nucleus (E) & 16.89 & 1.68 & -0.75 \\
W nucleus (W) & 17.80 & 0.68 & 0.10 \\
Jet (J) & 17.04 & 1.27 & -0.28 \\
Blue comp. (C) & 19.05 & -0.07 & -0.05 \\
Whole system & & 0.60 & 0.50
\end{tabular}

At the location of the nuclei several components show up in both the direct $B, V, R$ pictures and the residual ones after subtracting the PSF profiles (see CV). Figure $1_{a}$ shows the "total" image, obtained by coadding the three frames; fig $1_{b}$ shows the $\mathrm{V}$ residual image. The principal results, summarized in Table 1, are as follows: -1 . E has a large $V$ excess, which can be attributed to its strong $[O I I I]$ emission because the $[O I I I]_{5007}$ line falls in the $\mathrm{V}$ band.

- 2 . W has a stronger continuum and colors resembling those of a normal nucleus.

- 3. Jet-like feature $J$ extends $\sim 1.2^{\prime \prime}$ south of $E$. However, its $\mathrm{V}$ excess is smaller, suggesting a smaller contribution of emission lines in its spectrum, but excluding a pure continuum. $\mathrm{J}$ was discovered on HST images by Uomoto et al. (1993), who suggested that it may be scattered light from the obscured Seyfert 1 nucleus.

- 4. Fuzzy component $C$ is detected at $0.80^{\prime \prime} \pm 0.05^{\prime \prime}$ west of $W$. It has very blue colors, suggestive of a region of active star formation.

- 5. A "filament" connecting the 2 nuclei is faintly recognizable in all three frames but clearly visible in the "total" image (fig. $1_{a}$ ).

-6. Large tidal tails (Hutchings and Neff, 1989) are a conspicuous feature in the $R$ image.

- 7. $E$ and $J$ can be easily identified on the $B-V$ color map. The blue region around $\mathrm{W}$ seems to be connected to a knotty spiral arm to the north.

- 8. A linear structure centered on $\mathrm{E}$, with a PA $\sim 185^{\circ}$ and extending by $\sim 11.5^{\prime \prime}$ $S$ and $\sim 7^{\prime \prime} N$, can be seen on the $V-R$ color map. This must be primarily $[O I I I]$ emission (see below) and looks like an elongated ring.

-9. The projected separation between $\mathrm{E}$ and $\mathrm{W}$ is $3.98^{\prime \prime} \pm 0.02^{\prime \prime}$ at $\mathrm{PA}=83^{\circ}$.

\subsection{Spectroscopy}

Reconstructed images : - 1. Both nuclei are point-like in continuum light, embedded in a bright region elongated $\mathrm{E}$-W over many fibres. $\mathrm{E}$ is definitely redder than $W$; its reddening has been attributed to the presence of dust locally (Hutchings and Neff 1989).

- 2. The $H \alpha$ and $[O I I I]_{5007}$ emission images mainly show the linear structure in almost $\mathrm{N}$-S direction $\left(\mathrm{PA} \sim 182^{\circ}\right.$ ) centered on the $\mathrm{E}$ nucleus. It extends from $12.5^{\prime \prime} \mathrm{S}$ (edge of our field) to $\sim 7^{\prime \prime} \mathrm{N}$ (twisting eastwards).

Ionization maps Several regions, denoted with different symbols, can be identi- 
fied on the "ionization maps". Fig. $2_{a}$ shows as an example the $\log [O I I I]_{5007} / H \beta$ map, superposed on contours of the $[O I I I]_{5007}$ image. In Figure $2_{b}$ we display the $\log [O I I I]_{5007} / H \beta$ vs $\log [N I I]_{6583} / H \alpha$ diagram (using the same symbols). The main points are (for an extensive discussion see $\mathrm{CV}$ ):

- 1. The E nucleus (squared asterisk) has a typical Seyfert 2 spectrum, well explained by a power-law photoionization model (Ferland and Netzer, 1983). The index deduced from the $[\mathrm{HeII}]_{4686} / \mathrm{H} \beta$ ratio is rather steep and the ionization parameter $U$ decreases progressively with distance.

-2. The W nucleus (asterisk) falls clearly on the AGN side of the diagrams, at the limit between Seyfert 2 and LINERs. Unfortunately, the $[H e I I]_{4686}$ line is too noisy for a firm conclusion on the ionization mechanism.

- 3. For the region between the two nuclei (squares) and between the central regions and the southern emission knot (triangles) the data points follow the shock-wave model of Binette et al. (1985) very well. Shock-heating in this region might be due to interaction of the radio-jet $\left(18^{\prime \prime} S\right)$ with the ambient gas. - 4. The surprisingly high ionization in the emission knot $\sim 12-14^{\prime \prime}$ to the $S$ (crosses) might indicate either ionizing radiation from the central source collimated along the path of the radio jet, or photoionization by a local source of continuum (Binette et al. 1993 and references therein).

- 5 . In the region of the sharp ionization gradient, $\sim 3^{\prime \prime} \mathrm{N}$ of the nuclei (diamonds), both power-law photons and shocks might be collectively responsible for the ionization.

- 6. The northern end of the emitting region (X symbols) coincides with a spiral arm and its location on the diagnostic diagrams corresponds to HII regions.

Velocities and profile parameters: As an example, figure $3_{a}$ displays the spatial distribution of the $[O I I I]_{5007}$ (normalized) profiles (the reference velocity $\mathrm{V}_{0}=$ $15020 \mathrm{~km} \mathrm{~s}^{-1}$ is indicated) and figure $3_{b}$ displays the $80 \%$ velocities as shifts from $V_{0}$ (the regions of blue-asymmetric profiles are delimited by solid lines).

We found that: $\bullet 1$. A strong SW-NE velocity gradient occurs $\sim 6^{\prime \prime}$ around the $\mathrm{E}$ nucleus. It is $\sim 450 \mathrm{~km} \mathrm{~s}^{-1}$ for the $80 \%$ [OIII] velocities and somewhat less for the $50 \%$ velocities, with the steepest decrease $\left(\sim 250 \pm 50 \mathrm{~km} \mathrm{~s}^{-1}\right)$ within only $\sim 2^{\prime \prime} \mathrm{NE}$ of $\mathrm{E}$.

-2. Outside of this region the velocity field is smooth with $V_{80 \%} \simeq 15170 \pm 50$ $\mathrm{km} \mathrm{s}^{-1}$. The $E$ nucleus is blueshifted with respect to the $\mathrm{W}$ by $\sim 50-100 \mathrm{~km}$ $\mathrm{s}^{-1}$ at the $80 \%$ level of the different lines.

- 3. The $20 \%[O I I I]_{5007}$ asymmetry distribution does not quite match that of the velocities. The profiles are very asymmetric in the region of the velocity gradient $\left(A I_{20}= \pm 0.38\right)$, the sign of the asymmetry changing twice in this region. In the outer regions the asymmetries become much smaller.

- 4. The broadest $[O I I I]_{5007}$ lines are detected NE of the E nucleus; the $H \alpha$ line is broad on the $E$ nucleus and decreases outwards to become unresolved.

- 5. The apparent substructure in the profiles might reflect different components of emitting gas. In particular, the region of very blue-shifted, red-asymmetric, broad $[O I I I]_{5007}$ profiles might be associated with the $4^{\prime \prime} \mathrm{N}$ radio source: a blue component in the profile, due to bowshocks driven by the radio-jet into the ambient gas, could account for these effects (Taylor et al., 1992). In CV we examine the profiles along the radio/emission axis and suggest that the presence of at least three components, together with large amounts of dust in the central regions, would be needed in order to explain the complexity of the profiles. 


\section{Discussion}

S.Veilleux: Do you see correlations between the line widths in the external features and the strength of the low ionization lines, $[O I] / H \alpha$ and $[S I I] / H \alpha$ ? This might indicate that shock ionization is important in these features, perhaps due to interaction between the outflowing jet material and the ambient gas.

E.Chatzichristou: Yes, indeed, in the region located $\sim 3-4^{\prime \prime} \mathrm{N}$ of the nuclei, the $[N I I] / H \alpha$ and $[S I I] / H \alpha$ ratios become very strong and the $[N I I]$ profiles remarkably large. Unfortunately, the $[O I]$ line was not measurable in this region and the $[S I I]$ profiles were often blended and too noisy to allow systematic measurement of the line widths. However the above correlation seems real and could indicate shock ionization, the shocks being driven by the radio jet $4^{\prime \prime} \mathrm{N}$ of the $\mathrm{E}$ nucleus. The sharp velocity gradient with broadening of the [OIII] profiles, that we detected roughly in the same region, are indeed indicating important local motions.

R.Gelderman: It appeared that the FWHM of the $[O I I I]$ lines is largest where the $[O I I I] / \boldsymbol{H} \beta$ ratio is largest. Is this truly the case?

E.Chatzichristou: No, actually the $[O I I I]$ widths seem to correlate rather well with the regions of large velocity shifts.

A.Chrysostomou: Could the localized region of the low velocity you find be associated with the optical jet in any way?

E.Chatzichristou: No, they are in symmetrically opposite directions: the optical jet is located $\sim 1.2^{\prime \prime} \mathrm{SW}$ of the $\mathrm{E}$ nucleus, while the region of low velocities is detected $\sim 2-3^{\prime \prime} \mathrm{NE}$ of the $\mathrm{E}$ nucleus. However both must be associated with the symmetrical ejection of radio plasma from this nucleus.

\section{References}

Binette L., Dopita M., Tuohy I.R. (1985) ApJ 297,476

Binette L., Fosbury R.A., Parker D. (1993) PASP 105,1150

Chatzichristou E. and Vanderriest C. (1994) $A \& A$, submitted

Ferland G.J. and Netzer H. (1983) ApJ 264,105

Hutchings J.B. and Neff S.G. (1989) $A J$ 97,1306

Mazzarella J.M., Gaume R.A., Soifer B.T. et al. (1991) AJ 102,1241

Miller J.S. and Goodrich B.F. (1987) BAAS 19,695

Miller J.S. and Goodrich R.W. (1990) ApJ 355,456

Neff S.G.and Ulvestad J.S. (1988) $A J$ 96,841

Norman C. and Silk J. (1983) ApJ 266,502

Roos N. (198) $1 A \& A$ 95,349

Taylor D, Dyson J.E., Axon D.J. (1992) MNRAS 255,351

Uomoto A., Caganoff S., Ford H.C. et al. (1993) AJ 105,1308

Veilleux S. and Osterbrock D.E. (1987) ApJS 63,295 

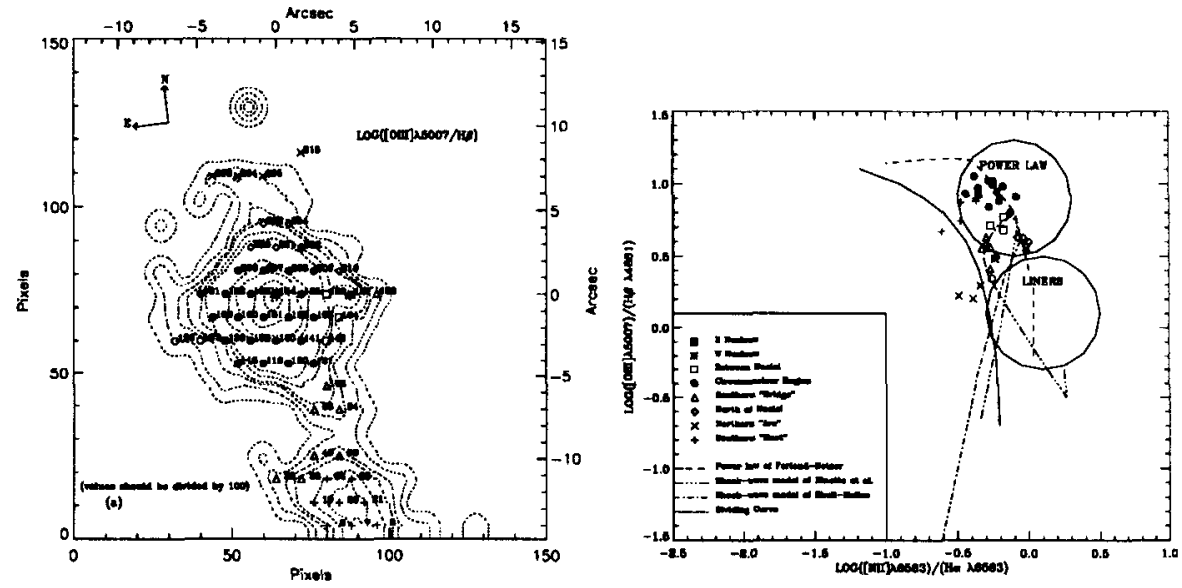

Figure 2. A typical "ionization map" and the corresponding diagnostic diagram(see text)

. A 14.4

(a) 11

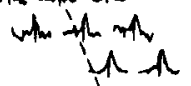
Nips $1-1-x$ rtw $\wedge \wedge \wedge \wedge \wedge \wedge-1$

$-1 \wedge \wedge \wedge \Lambda-1 \wedge-1$ 1. $\wedge \wedge$ - $1 \wedge \wedge-1$ $\wedge \Lambda-\Lambda i \Lambda-\Lambda$ $\wedge-1$ - Ni-1 $M-M a \Lambda \Lambda M$ wh

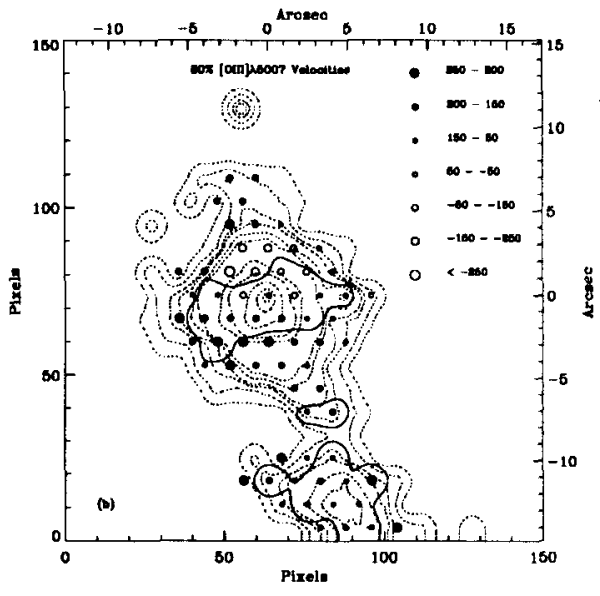

Figure 3. Some reconstructed maps for the $[O I I I]_{5007}$ emission line 